\title{
Constraint length inflation in fixed high rate convolutional codes and their impact on performance of O-IDMA
}

\author{
Ravi Prakash $^{1}$, Ajay Kumar Maurya ${ }^{2}$, Rakesh Kumar Maurya ${ }^{3}$, B. B. Tiwari ${ }^{4}$ \\ ${ }^{1,2,4}$ Department of Electronics Engineering Uma Nath Singh Institute of Engineering and Technology, \\ Veer Bahadur Singh Purvanchal University, India \\ ${ }^{3}$ Department of Electronics \& Instrumentation Engineering, Faculty of engineering and Technology, \\ Rohilkhand University, India
}

\section{Article Info \\ Article history: \\ Received Feb 10, 2020 \\ Revised Apr 7, 2020 \\ Accepted Apr 21, 2020 \\ Keywords: \\ Constraint length \\ Convolutional coder \\ Ex-OR gates \\ O-IDMA \\ Tree interleaver}

\begin{abstract}
To cope up with the higher data rate existing in modern communication systems multiple access technologies has been investigated OIDMA (Optical Interleave Division Multiple Access Technology) is one of the prominent technology use to fulfill this demand. Convolutional codes used in OIDMA systems enhance the performances and reduces the bit error rate of the system. In the present paper, the convolutional codes with increasing number of memory elements are used, the constraint length which depends on number of memory elements are increased with a systematic manner and their combined effect on response of OIDMA system has been observed. Tree interleavers in taken into account for analysis purpose and BER with increasing number of users is plotted in graphical and tabular manner.
\end{abstract}

Copyright $@ 2020$ Institute of Advanced Engineering and Science. All rights reserved.

\section{Corresponding Author:}

Ajay Kumar Maurya,

Department of Electronics and Instrumentation Engineering,

Veer Bahadur Singh Purvanchal University Jaunpur, Uttar Pradesh, India.

Email: ajaybtech84@gmail.com

\section{INTRODUCTION}

The multiple access schemes are frequently used in wireless $\&$ mobile communication to meet out the larger traffic intensity. Traffic intensity, measured in erlang is a tool to identify and count total quantity of users in a particular territory [1-2]. As traffic intensity is increasing multiple access interference (MAI) problem, inter-symbol interference (ISI) problem multiuser detection, near far problems are arising in existing multiple access technique like CDMA, TDMA, FDMA, DS-CDMA etc. [3-4]. To cope up heavy traffic intensity of users, solution is to use IDMA technology which drastically removers the all problems mentioned earlier in multiple access techniques.

The performance of CDMA-UWB signal is examined by using Gaussian pulses shape at optical source [5]. The performance of Multi Carrier Code Division Multiple Access (MC-CDMA) modulation using MERRY algorithm and provides the better performance as compaererd to OFDM system [6]. They described the BER performance in F-OFDM system using the $\mathrm{BCH}$ code over a multipath fading channel and fulfilled the requirement of 5G [7]. The authors also described the PAPR performance of F-OFDMA system using the Arithmetic coding and Huffman coding [8]. They evaluate the BER performance of OFDM systems using various channels (Fading, Rayleigh, Rician and AWGN) [9].

When optical technology is incorporated in IDMA it is more effective known as OIDMA. As we know that optical technology possesses advantages in terms of enormous potential bandwidth, low loss, less interference, low cross talk, minimum bit error rate which makes it preferable for longer distance transmission. IDMA is special form of CDMA where extension of bandwidth is done by same PNS code to all users while user's separation is achieved by specific interleavers for each user [10-12]. By using single 
spreading codes, problems of cross correlation among various spreading codes and multiusers interference problems are greatly reduced, bandwidth of IDMA is also compressed and more number of users is served in limited particular bandwidth. The multiuser detection at receivers is less complicated as compared to CDMA because receiver has no need to store the autocorrelation, cross-correlation, noise samples, normalized power of various users at the detector, but only measures long likely hood ratio (LLR), APP and recognize user on basis of these quantities [13-15].

Convolutional encoder for varying constraint length 3, 5, 7,9 and 11 is taken for analysis purpose. Fixing the code rate and adding the shift register with the systematic manner one by one convolutional encodersof different constraint length 3,5, 7,9 and 11are designed which produces more uncorrelated code-words at the output and enhances the error correcting capacity of code theoretically [16-17]. Due to this reason bit error rate be reduced. Using such type of convolutional encoder hardware connection in OIDMA system the quality and performance of OIDMA has been checked in the present article.

In this paper, Section 2 describes the block diagram of coded optical inter-leave division multiple access system (O-IDMA) and explains the process of tree interleaving. Convolutional coding, designing architecture of convolutional encoder by varying memory elements are explained in Section 3. Section 4 shows the simulation result and their discussion. Conclusion is discussed in Section 5.

\section{OVERVIEW OF OIDMA SYSTEM}

The architecture of OIDMA system using convolutional coding is shown in Figure 1. In the architecture section, the first component represents encoder. The encoder is able to reducing errors generated by a channel for any digital communication. Here, $\mathrm{k}^{\text {th }}$ number of encoder is used for encoding the $\mathrm{k}^{\text {th }}$ number of users, after encoding, the encoded data is spreaded with PNS sequence by spreader. After this the resulting signal data is more secure and almost free from interference, and transmitted to interleaver. The resulting signal is interleaved by using $\mathrm{k}^{\text {th }}$ number of interleavers. After this interleaved data is combined by combiner and transmitted through optical source (LASER), reached to receiver section [18-19].

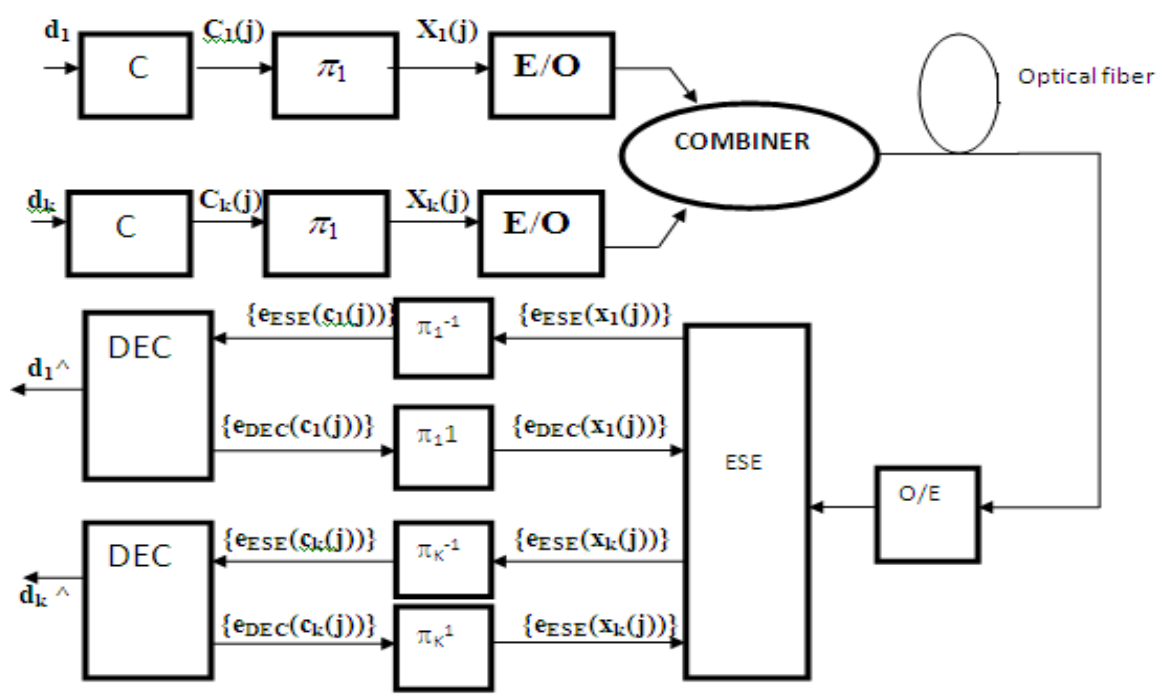

Figure 1. Optical IDMA transmitter and receiver structure

In receiver section, optical data is firstly converted by optical detector (APD) into electrical signal. The received signal is noisy signals; the noisy signal is decoded by chip-matched method for multiuser detection and passes to elementary signal estimator. By using ESE, LLR (Log Likelihood Ratio), and feedback mechanism is determines the specific users [20-21].

The mechanism of interleaver is shown in Figure 2. Here, tree interlever is used to reduce the large memory requirement and computational complexity [22-23]. The tree interleaver consist of the two master random interleavers $\Pi 1$ and $\Pi 2$ which are orthogonal to each other. The tree interleaver provides zero cross correlation and user-specific inter-leavers [23]. 


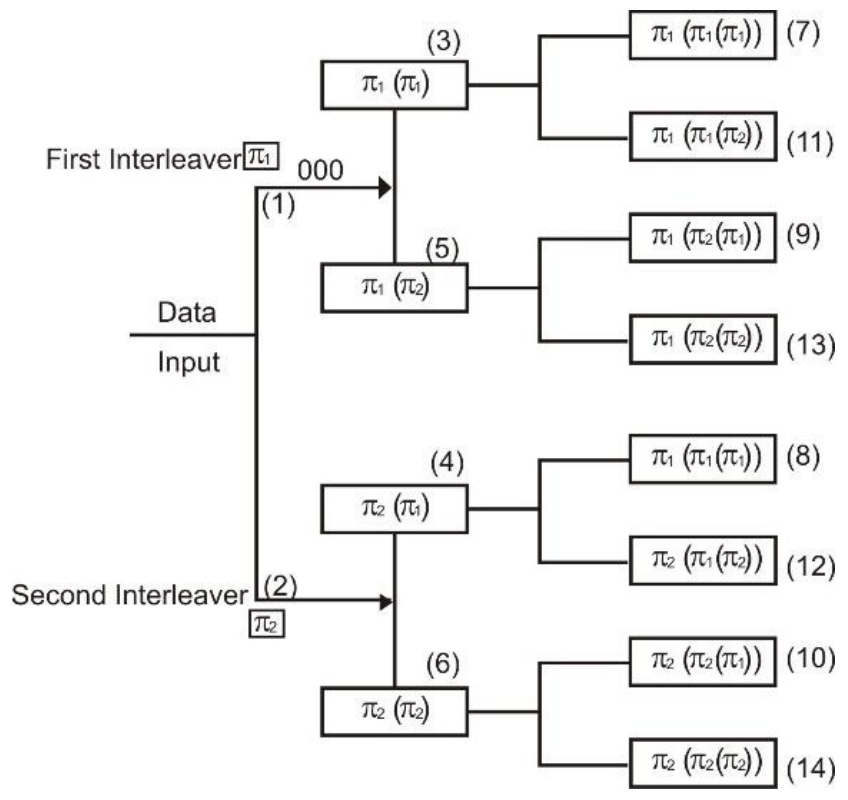

Figure 2. Tree based interleaving technique

\section{CONVOLUTIONAL CODING}

In digital communication, for error correction convolutional coding is used. The convolutional coding consist of the D flip-flops and logic gates. The shifting property of convolutional encoder is generally used to encoder hardware design. The D flip-flops used as the shift register, and the number of shift register decides the length of convolutional encoder which is known as constraint length whose variation produces uncorrelated code words [16-17].

The convolutional encoder are designed by three terms A, B and C, where A is known as number of output bits, $\mathrm{B}$ is number of input bits and $\mathrm{C}$ is number of memory elements. The code rate is known as [B/A]. When the number of Ex-OR gates increase, the output bits, $A$, also increases, and generate the code rate $1 / 2,1 / 3 \& 1 / 4$ etc. with a fixed number of input bits, $B$, fixed to 1 . The constraint length $\mathrm{L}=\mathrm{m}+1$, where $\mathrm{m}$ represents shift registers in use. When using higher number of shift register, output bits are highly influenced by a single bit [24-25].

\subsection{Architecture of convolutional encoder by varying memory elements}

In designing technology of convolutional encoders flip flops decides the constraint length of encoder [17].In the Figure 3 to Figure 7 shown below there are five convolutional encoders are designed for fixing the Ex-OR gates and different constraint lengths [26-27].In present work initially we have taken two memory elements with constraint length $\mathrm{L}=3$ \& fixing the number Ex-OR gates 4 , the feedback connections are decided by trellis [5 77 7] in octal representation form [101, 111, 111, 111]. This is the basic network topology of convolutional encoder having code rate [1/4] shown in Figure 3. Now by fixing the Ex-OR gates four then number of memory elements are increasing $4(\mathrm{~L}=5) \& 6$ that is $(\mathrm{L}=7)$ respectively shown in Figure 4 and Figure 5. In Figure 4 for $(1,4)$ code rate possible optimum trellis are 25273337 in octal number representation [010 101, 010 111, 011 011, 011 111] which decides the feedback mechanism of encoder. Similarly, in Figure 5, L is increased as 7 that is 6 memory elements are connected with code rate 1/4 and possible trellis are selected as [135 135147 163] that is [001 011 101, 001011101,001100111 , $001110011]$ in octal number and encoder feedback are setting accordingly.

Similarly, now fixing the Ex-OR gates four then number of memory elements are increasing 8 $(\mathrm{L}=9) \& 10$ that is $(\mathrm{L}=11)$ respectively shown in Figure 6 and Figure 7 . In Figure 6 for $(1,4)$ code rate possible optimum trellis are 463, 535, 733, 745 in octal number representation [100 110011,101011101 , 111011011,111100 101] which decides the feedback mechanism of encoder. In Figure 7, L is increased as 11 that is 10 memory elements are connected with code rate $1 / 4$ and possible trellis are selected as [2327 23532671 3175] that is [010 $011010111,010011101011,010110111001,011001111101]$ in octal number and encoder feedback are setting accordingly. The optimum trellis [23, 35] are taken from [reference-27 convolution coder chapter-10] which contains tabular form of all possible trellis combination for different code rates and constraint lengths as shown in Table 1. Theoretically as the number of memory elements is increasing accuracy and error detecting capability should be increased, but due to increasing number of flip flops delays of flip flops might come in pictures which decreases the speed of system. 


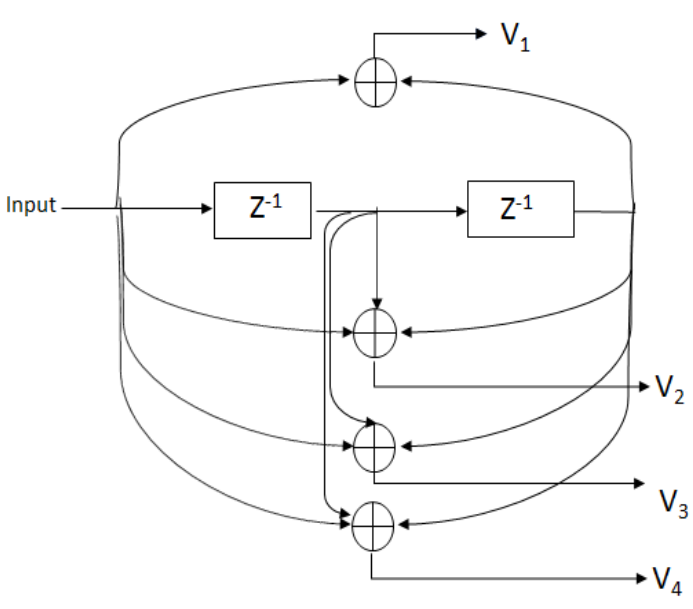

Figure 3.Convolutional encoder [1, 4] for constraint length $\mathrm{L}=3$

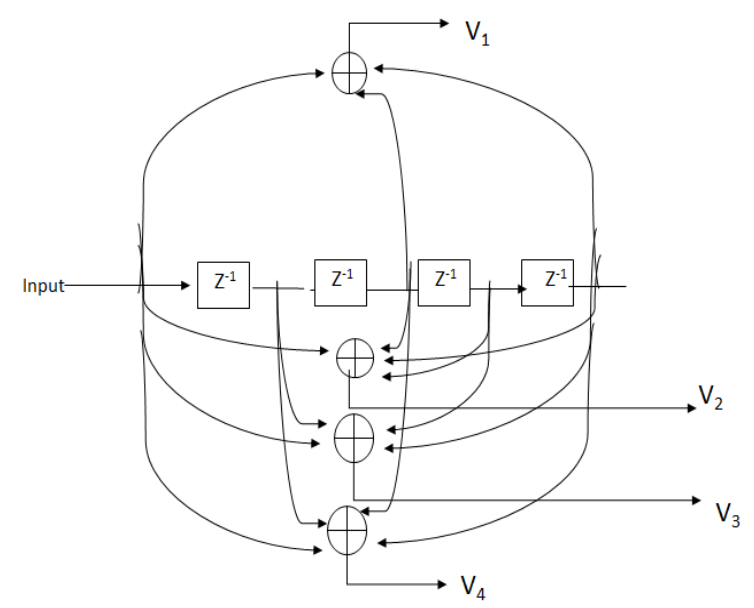

Figure 4.Convolutional encoder [1, 4] for constraint length $\mathrm{L}=5$

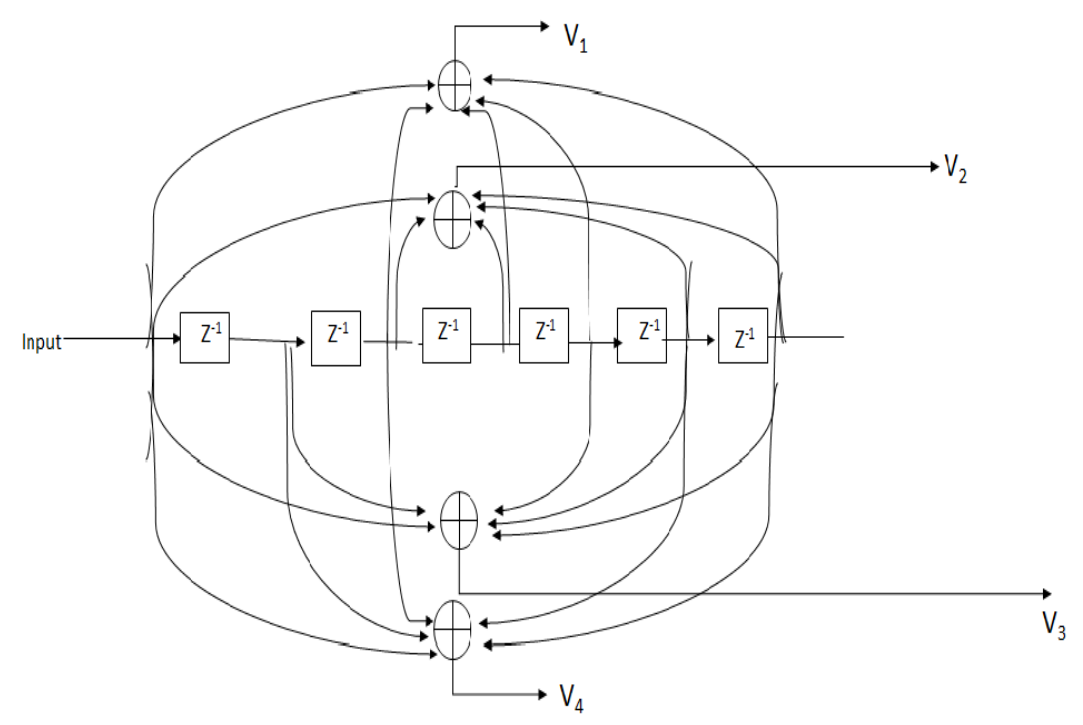

Figure 5.Convolutional encoder [1, 4] for constraint length $\mathrm{L}=7$

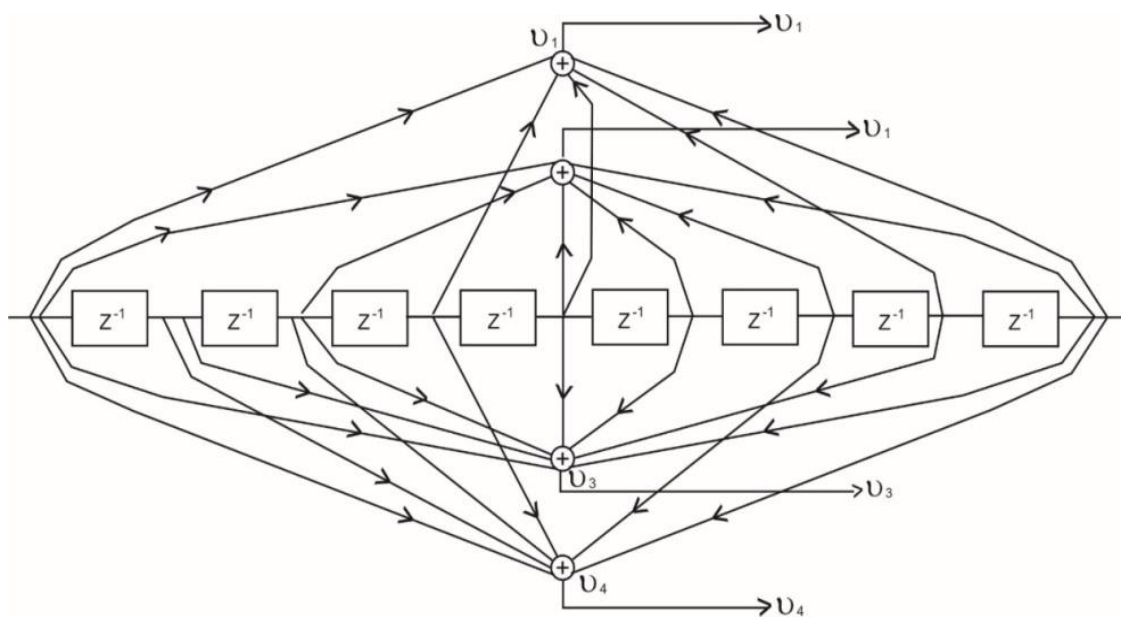

Figure 6.Convolutional encoder [1, 4] for constraint length $\mathrm{L}=9$ 


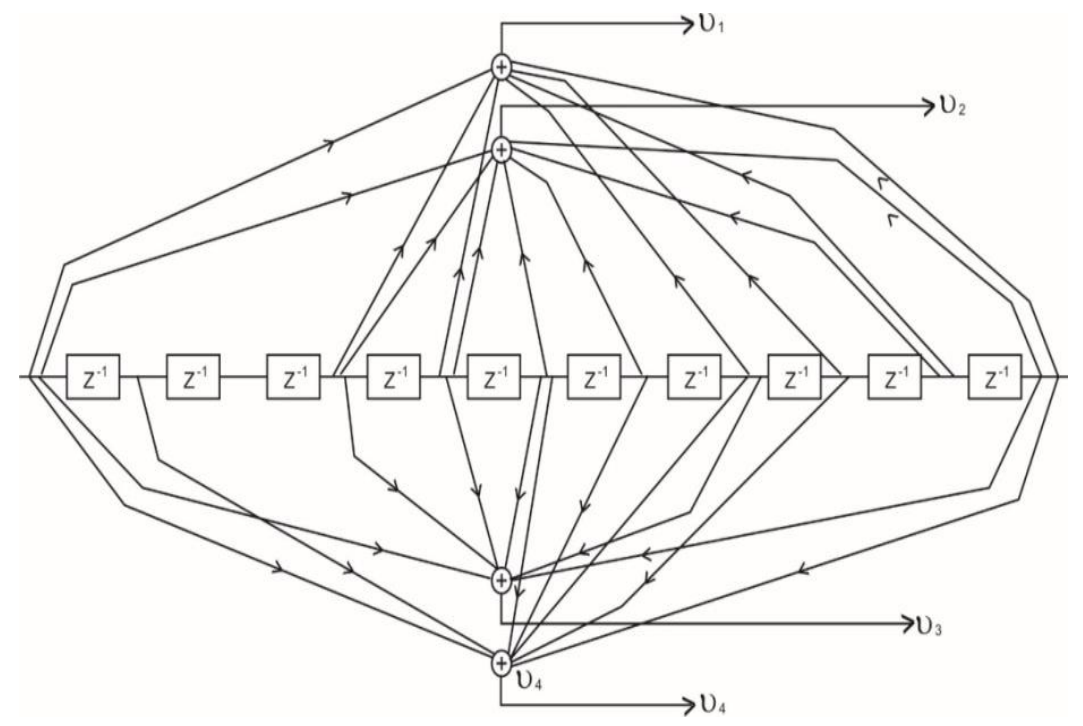

Figure 7. Convolutional encoder $[1,4]$ for constraint length $\mathrm{L}=11$

Table 1.Maximum freedistancecodes [code rate 1/4][Source - Larsen (1973)]

\begin{tabular}{ccc}
\hline Constraint Length/ $\mathrm{K}$ & Generators in Octal $\mathrm{G}_{1} \mathrm{G}_{2} \mathrm{G}_{3} \mathrm{G}_{4}$ & $\mathrm{~d}_{\text {free }}$ \\
\hline 3 & $(5,7,7,7)$ & 10 \\
5 & $(25,27,33,37)$ & 16 \\
7 & $(135,135,147,163)$ & 20 \\
9 & $(463,535,733,745)$ & 24 \\
11 & $(2337,2353,2671,3175)$ & 29 \\
\hline
\end{tabular}

\section{SIMULATION RESULT AND DISCUSSION}

The OIDMA system with various blocks are programmed and interfaced to each other. The constraint length of convolutional codes is varied from $\mathrm{L}=3$ to $\mathrm{L}=11$ means memory elements are changed as 2, 4, 6, 8, 10 respectively with fixed four number of Ex-OR gates. Since the number of Ex-OR gates are set as 4 the code rate is $(1,4)$ which is termed as high code rate. The network topologies of coders are designed with the help of [Reference Paper 28, Conv. Codes paper chapter 10]. This paper deals with the constraint length and their respective generators in octal for fixed code rates. The network topology isdesigned according to $\mathrm{G}_{1} \mathrm{G}_{2} \mathrm{G}_{3} \mathrm{G}_{4}$ values assigned for code rate $(1,4)$.

The various topologies are connected in coding section of OIDMA system. The inter-leaver is selected as tree inter-leaver. At the input parameters the spread length is set as 16, block length 100 and data length 512 is fixed. At the optical parameter the Gaussian pulse of $1 \mathrm{~mW}$ power, maximum rate $1000 \mathrm{Mbps}$ with fixed fiber numerical aperture and dispersion parameters, APD as on optical detection with gain 1000 is selected, the operating wavelength is $1553 \mathrm{~nm}$. At the input number of users are changed starting from 220 users up to 340 users. The observed BER for various constraint lengths are tabulated and the graph is plotted in Table 2 and Figure 8.

Table 2. B.E.R. performance for various constraint length $(\mathrm{L}=3,5,7,9$ and 11$)$ with fixed code rate $(1,4)$

\begin{tabular}{|c|c|c|c|c|c|}
\hline $\begin{array}{l}\text { No. of } \\
\text { Users }\end{array}$ & $\begin{array}{c}\text { BER for } \\
\text { Constraint Length } \\
\text { L }=3\end{array}$ & $\begin{array}{c}\text { BER for } \\
\text { Constraint Length } \\
\text { L }=5\end{array}$ & $\begin{array}{c}\text { BER for } \\
\text { Constraint Length } \\
\text { L }=7\end{array}$ & $\begin{array}{c}\text { BER for } \\
\text { Constraint Length } \\
\text { L }=9\end{array}$ & $\begin{array}{c}\text { BER for } \\
\text { Constraint Length } \\
\mathrm{L}=11\end{array}$ \\
\hline 200 & No Error & No Error & No Error & No Error & No Error \\
\hline 220 & $1.2480 \times 10^{-9}$ & $0.7248 \times 10^{-9}$ & $6.4210 \times 10^{-10}$ & $1.3450 \times 10^{-10}$ & No Error \\
\hline 240 & $2.3562 \times 10^{-9}$ & $1.1956 \times 10^{-9}$ & $0.9465 \times 10^{-9}$ & $0.3468 \times 10^{-9}$ & $9.3750 \times 10^{-11}$ \\
\hline 260 & $3.9240 \times 10^{-9}$ & $2.6483 \times 10^{-9}$ & $1.1102 \times 10^{-9}$ & $0.6240 \times 10^{-9}$ & $3.3450 \times 10^{-10}$ \\
\hline 280 & $5.2138 \times 10^{-9}$ & $3.4240 \times 10^{-9}$ & $2.4832 \times 10^{-9}$ & $1.3450 \times 10^{-9}$ & $0.9501 \times 10^{-9}$ \\
\hline 300 & $6.1240 \times 10^{-9}$ & $4.3925 \times 10^{-9}$ & $3.5368 \times 10^{-9}$ & $2.3260 \times 10^{-9}$ & $1.3875 \times 10^{-9}$ \\
\hline 320 & $9.3250 \times 10^{-9}$ & $7.3400 \times 10^{-9}$ & $4.3620 \times 10^{-9}$ & $3.1342 \times 10^{-9}$ & $1.9240 \times 10^{-9}$ \\
\hline 340 & $1.1243 \times 10^{-8}$ & $9.3905 \times 10^{-9}$ & $6.3442 \times 10^{-9}$ & $5.2405 \times 10^{-9}$ & $3.2432 \times 10^{-9}$ \\
\hline
\end{tabular}




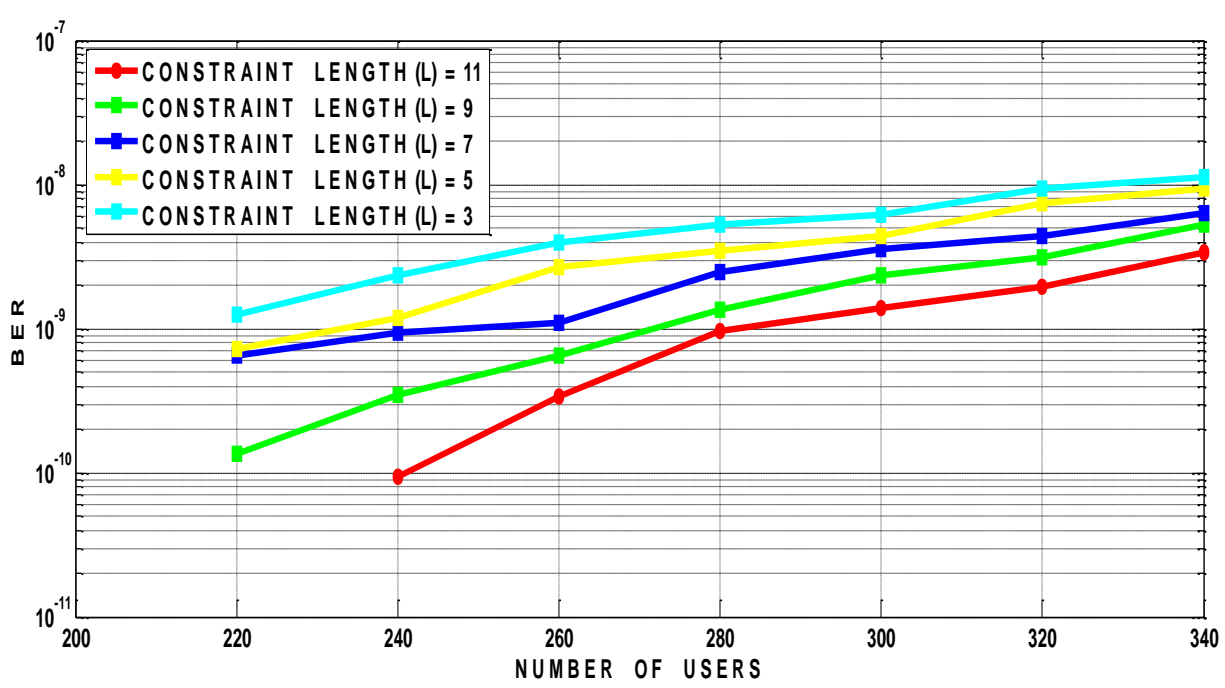

Figure 8.B.E.R. performance for various constraint length $(\mathrm{L}=3,5,7,9$ and 11$)$ with fixed code rate $(1,4)$

From the observed reading obtained for $\mathrm{L}=3$, clearly shows that BER is $1.2480 \times 10^{-9}$ for 220 users which is increasing with number of users and reaches up to $1.1243 \times 10^{-8}$ for 340 users. It clearly similar to the theoretical aspects that as the users increases more MAI introduces which enhances BER. Some trend is obtained for all cases of constraint length from $\mathrm{L}=3$ to $\mathrm{L}=11$. As the number of memory elements increases from 2 to 4 the readings of BER is reduced from $1.2480 \times 10^{-9}$ to $0.7248 \times 10^{-9}$ (220 user) which further reduces up to $6.4210 \times 10^{-10}$ (220 user) for memory element 6 . This gradual reduction in BER resembles with theoretical aspects that using more memory elements in coder increases the $d_{\text {free }}$ which is cause of reduction in BER. The best reading of BER obtained for the case when constraint length $=11$, memory element 10 with values $9.375 \times 10^{-11}$ for 240 users.

\section{CONCLUSION}

From the results obtained clearly indicates that using tree inter-leaver in OIDMA with various network topologies of convolutional encoder connected the readings of BER is quiet good and reaches up to $10^{-11}$. The best architecture designed when ten memory element with proper generators in octal 2337, 2353, 2671,3175 are connected andthe performance improvement is phenomenal. As we increase the number of memory elements more than ten results might be more improved but system might be slowly because the combined delay produced by all flip flops comes into picture which affects the speed. Overall we conclude that by setting a proper number memory element with suitable connection of convolutional encoder connected in OIDMA will be a best alternative among all existing multiple access techniques to support enormous data rate.

\section{REFERENCES}

[1] Z. Pan, et al.,"Optical performance monitoring for the next generation optical communication networks,"Optical Fiber Technology, vol. 16, no. 1, pp. 20-45, 2010.

[2] A. Shukla,et al., "Multiple Access Scheme for Future (4G) Communication, A Comparison Survey,"International Symposium on Devices MEMS, Intelligent Systems \& Communication (ISDMISC), vol. 1, 2011.

[3] A. K. Dutta, "Optimum Detection of CDMA,"International Journal of All Research Education and Scientific Methods (IJARESM), vol. 4, no. 3, pp. 1-5, 2016.

[4] J. A. Salehi, "Code division multiple-access techniques in optical fiber networks-Parts I: Fundamental principles," IEEE Transactions on Communication,vol. 37, no. 8, pp. 824-833, 1989.

[5] C. G. Chin, et al., "Bit-error-rate Optimization for CDMA Ultra-wideband System Using Generalized Gaussian Approach," International Journal of Electrical and Computer Engineering (IJECE), vol. 7, no. 5, pp. 2661-2673,2017.

[6] F. Bouasria, et al., "A blind channel shortening for multiuser, multicarrier CDMA system over multipath fading channel," TELKOMNIKA Telecommunication Computing Electronics and Control, vol.17, no.4, pp.1692-1697, 2019.

[7] G. A. Hussain and L. Audah, "BCH codes for 5G wireless communication systems over multipath fading channel," Indonesian Journal of Electrical Engineering and Computer Science, vol. 17, no. 1, pp. 310-316, 2020.

[8] A. Idris, et al., "PAPR Reduction Using Huffman and Arithmetic Coding Techniques in F-OFDM System,"Bulletin of Electrical Engineering and Informatics, vol. 7, no. 2, pp. 257-263, 2018. 
[9] P. Manhasa and M. K. Soni, "Performance of OFDM System under Different Fading Channels and Coding," Bulletin of Electrical Engineering and Informatics, vol. 6, no. 1, pp. 54-61, 2017.

[10] Y. Lin, et al., "Design and Analysis of Asynchronous Incoherent Optical-CDMA Systems Using a New CodeShifting Technique," IEEE Transactions on Communications, vol. 63,no. 7, pp. 2619-2631, 2015.

[11] K. Kumaravel, "Comparative Study of 3G and 4G in Mobile Technology,"IJCSI International Journal of Computer Science Issues, vol. 8, no. 5, pp. 256-263, 2011.

[12] A. Basharat, et al., "CDMA versus IDMA for Subscriber Cell Density,"2008 International Conference on Innovations in Information Technology, pp. 520-524, 2008.

[13] T. K. Alumona, et al., "Analysis of DSSS - CDMA System for Varying Number of Users," Computer Engineering and Intelligent Systems, vol. 6, no. 11, pp. 11-14, 2015.

[14] A. M. Morsy, et al., "Performance Analysis and Comparision of optical IDMA and optical CDMA Techniques using Unipolar Transmission Scheme," 18th European Confrence on network and optical communication, pp. 55-60, 2013.

[15] L. Ping, et al., "Recent Progress in Interleave-Division Multiple-Access (IDMA),"MILCOM 2007 - IEEE Military Communications Conference, pp. 1-7, 2007.

[16] S. V. N. Reddy, et al., "Design of Convolutional Codes for varying Constraint Lengths," International Journal of Engineering Trends and Technology, vol. 4, no. 1, pp. 61-66, 2013.

[17] S.V. Viraktamath, "Impact of constraint length on performance of Convolutional CODEC in AWGN channel for image application," International Journal of Engineering Science and Technology, vol. 2, no. 9, pp. 4696-4700, 2010.

[18] P. Bhulania, et al., "Issues in Analysis \& Design of IDMA \& its Application," International Journal of Advanced Research in Electrical, Electronics and Instrumentation Engineering, vol. 2, no. 5, 2013.

[19] Y. H. Chung, "Performance and Bandwidth Efficient Interleave-Division Multiple Access Scheme with HighSpread Interleavers," 2007 6th International Conference on Information Communications and Signal Processing, pp. 1-5, 2007.

[20] F. Begum, "Implementation of Interleave Division Multiple Access (IDMA) with Multiple Users in Wireless Communication System," International Journal of Computer Applications, vol. 134,no.15, 2013.

[21] L. Ping, "Interleave Division Multiple Access and chip by chip Iterative multi user Detection," IEEE Communication Magazine,vol. 43, no. 6, pp. 19-23, 2005.

[22] M. Gupta, et al., "Comparative study of Random and Tree Base Interleaver for Optical IDMA," Fourth International Conference on ComputationalIntelligence and Communication Networks, pp. 271-275, 2012.

[23] M. Shukla, et al.,"Analysis and Design of Tree Based Interleaver for Multiuser Receivers in IDMA Scheme,” 208 16th IEEE Conference on Networks,pp. 1-4, 2008.

[24] E.R. Berlekamp, "The technology of error-correcting codes," Proceedings of the IEEE, vol. 68, no. 5, pp. 564-593, 1980.

[25] G. C. Clark and J.B. Cain, "Error correction coding for digital communications,"Perseus Publishing, 1981.

[26] “LECTURE 8:Convolutional Coding,” MIT 6.02 DRAFT Lecture Notes Fall 2010, pp. 1-8, 2010.

[27] C. P. Li,"Maximum Free Distance Codes in Chapter 10 Convolutional codes,"Wireless Information Transmission Sytem Lab., Institute of Communications Engineering National Sun Yat - Sen University.

\section{BIOGRAPHIES OF AUTHORS}

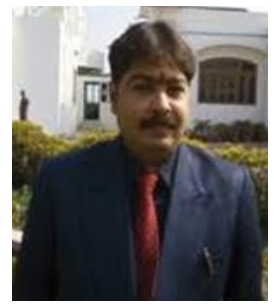

Ravi Prakash is working as an assisastant Professor in Electronics and Communication Engneering Uma Nath Singh Institute of Engineering and Technology Veer Bahadur Singh Purvanchal University Jaunpur, Uttar Pradesh, India. He was born on January 19, 1975, in Jaunpur, U.P., India. He received the B. Tech. degree in Electronics and Telecommunication Engineering from University of Allahabad, Allahabad, U.P., India in 1997, and M. Tech. degree in Electronics Engineering (Communication Technology) from University of Allahabad, Allahabad, U. P., 2001. He had worked as head of department of Electronics Engineering at Veer Bahadur Singh Purvanchal University Jaunpur for almost ten years. He is currently working the D.Phil degree in Electronics and Communication Engineering from University of Allahabad, U.P., India. His current research interests include Communication Technology. He has published several research articles in reputed international journals related to wireless, optical and digital communication.

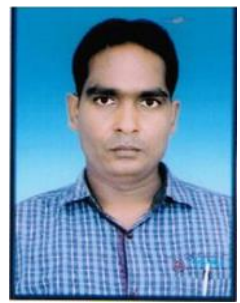

Ajay Kumar Maurya is working as an assisastant Professor in Electronics and Instrumentation Engneering Uma Nath Singh Institute of Engineering and Technology Veer Bahadur Singh Purvanchal University Jaunpur, Uttar Pradesh, India.Hewas born on October 31,1986, in Jaunpur, U.P., India. He received theB.Tech degree in Electronics \&Communication Engineering from V.B.S.Purvanchal University Jaunpur, U.P., India in2008, and M.Tech degree in Digital Communication fromBundelkhand Institute of Engineering and Technology Jhansi,U.P., India in 2011. He is currently working the Ph.D.degree in Electronics Engineering from Kamla Nehru Instituteof Technology Sultanpur, U.P., India. His current researchinterests include Communication Technology and wireless communication. He has been teaching in Veer Bahadur Singh Purvanchal University since last ten years and published approximately 20 research article in reputed journals. 

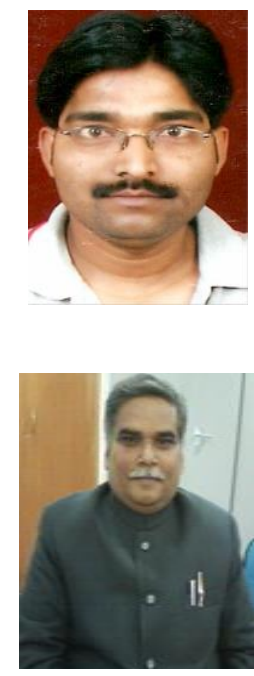

Dr. Rakesh k. Maurya was born in Jaunpur, U.P, India in 1975. He received his B.Tech (Electronics \& Instrumentation Engg.) degree from Rohilkhand University, Bareilly in 2000, M.E. (Instrumentation \& Control) degree from NITTTR, Chandigarh, in 2009, and Ph.D (Electronics Engineering) from U.K. Technical University Dehradun in 2017. From 2002 to 2008 he was a lecturer, 2008 to 2013 he was an Assistant Prof. (AGP-7000), 2013 to 2016 he was an Assistant Prof. (AGP-8000) and currently he is Associate Prof. (AGP-9000) at the Department of Electronics and Instrumentation Engineering MJP, Rohilkhand University, Bareilly. He is working in the area of Microprocessor and Microcontroller, Neural Network, Fuzzy inference System, and their applications to microstrip antennas.

Prof. B.B.Tiwari graduated from the then University of Roorkee, Roorkee in 1986 in Electronics and Communication Engineering. He served at the Thapar University of Engineering and Technology Patiala, IET Lucknow, Dr. RML Avadh University, Ayodha and rose to the position of Professor at VBS Purvanchal University, Jaunpur in 2001. On lien from VBS Purvanchal University, he served as a Professor in Guru Jambheshwar University of Science and Technology, Hisar and IIIT Allahabad as well. Currently he is professor and HOD, Department of Electronics Engineering at VBS Purvanchal University, Jaunpur. Prof. Tiwari worked with two prestigious projects with the GOI: one with AICTE New Delhi -a Research and Development project worth a sum of Rs. 6.00 Cr. grant from DRDO to develop beam combiner for the head up display in fighter planes. Currently he is discharging the duty of Director/ Coordinator-TEQIP-III project from World Bank for Uma Nath Singh Institute of Engineering and Technology, a faculty of VBS Purvanchal University worth Rs. $10.00 \mathrm{Cr}$ initially but likely to enhance further. He has guided/continuing to guide Ph.D scholars and organized national and international conferences/workshops etc. He has published 110 research papers in the international/national journals and proceedings of conferences. He has delivered lectures on topics of varied disciplines across the country. He has attended as well as organized short term course, FDPs, EDPs, and PDTs, at IITs/IISC/IIMs/Industries etc. He is fellow of IETE (I), IE (I), member ISTE, and Member IEEE (USA) etc.He is Director at the Institute of Pharmacy at Jaunpur and Member of the executive council of the University. He has been Dean, Faculty of Engineering and Technology for 2 terms at Jaunpur as well as Director of the same institute for 5 years. Professor Tiwari has written a book on 'Signal and Noise' in Communication Engineering. 